Cinémas

Revue d'études cinématographiques

Journal of Film Studies

\title{
Ah! les salauds!
}

\section{Jean-Louis Leutrat}

Volume 4, numéro 3, printemps 1994

Questions sur l'éthique au cinéma

URI : https://id.erudit.org/iderudit/1001038ar

DOI : https://doi.org/10.7202/1001038ar

Aller au sommaire du numéro

Éditeur(s)

Cinémas

ISSN

1181-6945 (imprimé)

1705-6500 (numérique)

Découvrir la revue

Citer cet article

Leutrat, J.-L. (1994). Ah! les salauds! Cinémas, 4(3), 73-84.

https://doi.org/10.7202/1001038ar

\section{Résumé de l'article}

Cet article porte sur le film Allemagne 90 de Jean-Luc Godard. Il met en valeur l'éthique à l'oeuvre dans ce film, résidant dans l'entremêlement des combats, le " combat-contre » et le " combat-entre » (Deleuze). Il conclut que le cinéaste, au moyen de son film, montre la dissimulation qui apparait, traque le moment de l'apparition. 


\section{Ah! les salauds!}

\section{Jean-Louis Leutrat}

\section{RÉSUMÉ}

Cet article porte sur le film Allemagne 90 de Jean-Luc Godard. Il met en valeur l'éthique à l'œuvre dans ce film, résidant dans l'entremêlement des combats, le "combat-contre" et le "combat-entre" (Deleuze). Il conclut que le cinéaste, au moyen de son film, montre la dissimulation qui apparaît, traque le moment de l'apparition.

\section{ABSTRACT}

This article concerns Jean-Luc Godard's film Allemagne 90. It highlights the ethics at work in this film, found in the intermixing of combats - the "combatagainst" and the "combat-between" (Deleuze). It concludes that the filmmaker, through his film, reveals the dissimulation that appears and traces the moment of its appearance.

Quand tout s'est obscurci, règne l'éclairement sans lumière qu'annoncent certaines paroles.

Maurice Blanchot, L'Écriture du désastre

Plus d'une fois le visage crevassé d'un homme au bord de la mort a occupé un écran de cinéma ou de télévision. L'acteur, car il s'agit de lui autant que du personnage, est à la fin de sa vie, épuisé. C'est sa dernière apparition. Quelques mois plus tard, il ne sera plus. Il a beaucoup vécu, ses yeux sont enchâssés dans les plis d'une chair ravinée par les expériences, la succession des 
jours qui arrive à son terme, et vraisemblablement, l'alcool. Eddie Constantine, dans Allemagne 90 Neuf Zéro de Jean-Luc Godard, rappelle non seulement celui qu'il fut dans Alphaville, mais aussi Buster Keaton tel que le montrait Film de Samuel Beckett et Alan Schneider. Coöncidence, ces deux œuvres datent de 1965. Film porte le deuil des années vingt, quand le burlesque et tout le cinéma (par conséquent Keaton) étaient encore jeunes; l'acteur rase les murs d'une ville qui semble d'abord dévastée comme celle d'Allemagne année zéro (1948). Alphaville décrit un futur où les dictionnaires seront purgés et l'amour une vieillerie en passe d'être oubliée. L'idée de Godard est de faire revenir dans Allemagne 90 le personnage de Lemmy Caution en prenant en compte ce qu'a été l'Histoire des hommes et ce que le temps a inscrit sur le corps d'Eddie Constantine. En 1965, Keaton préfigurait Constantine et Alphaville, l'Allemagne des années quatre-vingt-dix. Allemagne 90 porte la trace de ce qui est plus qu'un regret et qui renvoie de nouveau aux années vingt, quand le communisme était lui aussi encore jeune, que l'on pouvait associer les mots Russie et Bonheur et qu'Eisenstein devenait Sa Majesté Eisenstein... Tel est le nœud d'intrications temporelles dont est constitué le film de Godard. À travers ce travail délicat sur le temps, l'Histoire et les corps, il est possible de voir s'exprimer une éthique.

Que faut-il entendre par là? D'abord, une attitude qui " [...] s'inscrit en faux contre l'indifférence, l'arbitraire et le laisser-aller " (Jacob, p. 875) et où, assez mystérieusement, le politique et le poétique se rejoignent. Plus que de porter un jugement, il s'agit d'entrer dans un combat, de créer pour ce combat, grâce à l'œuvre, la possibilité même d'exister. Il y a deux sortes de combats, comme l'explique Gilles Deleuze, le combat-contre et le combat-entre: "Il faut distinguer le combat contre l'Autre, et le combat entre Soi. Le combat-contre cherche à détruire ou à repousser une force $[\ldots]$, mais le combat-entre cherche au contraire à s'emparer d'une force pour la faire sienne. Le combat-entre est le processus par lequel une force s'enrichit, en s'emparant d'autres forces et en s'y joignant dans un nouvel ensemble, dans un devenir" (1993, p. 165). L'éthique, au sens où nous l'entendrons, réside donc dans l'entremêlement de ces combats. 
Allemagne 90 engage un combat contre ceux pour qui Karl Marx et le devenir révolutionnaire des hommes sont enterrés ("Heureux non-anniversaire»), que ce soit en Allemagne ou dans le monde, c'est-à-dire les représentants du capitalisme international, ses serviteurs, ses adorateurs, les Maîtres du Monde qui envisagent comme avenir sinon l'autodestruction de l'humanité, du moins son décervelage. Il engage aussi un combat contre l'oubli des camps de concentration et de ceux qui furent décapités à la hache, torturés, mutilés. Il engage un combat contre la germanisation des États-Unis et l'américanisation de l'Allemagne de l'Ouest, contre l'hégémonie du système américain considéré comme la menace la plus dangereuse dans notre monde, à commencer pour le cinéma. Telle est la part de discours non ambiguë du film, celle qui n'est pas par nature destinée à lui donner les qualités d'une grande œuvre, mais qui du moins le dote d'un point de vue et d'une dignité. Serge Daney dit que c'est un "[...] film habité. Sans creux, ni temps morts. Habité par son sujet...” (p. 359).

Un carton "Topographie de la terreur" résume cette dimension du film. L'expression demande explication, même si l'on peut deviner à quoi elle renvoie. En 1985, un groupe de Berlinois de l'Ouest s'émut auprès du maire de la ville de ce que le terrain où se trouvaient les organes de décision du III $^{c}$ Reich fût laissé à l'abandon ou menacé d'usages destinés à le banaliser. Les bâtiments qui occupaient ce périmètre important, fortement endommagés par les bombardements et les combats, avaient été pour la plupart détruits après la guerre. Même l'ancien musée d'ethnologie, restauré en partie et réutilisé comme musée de la préhistoire et de l'histoire ancienne, fut démoli en 1963. Sur ce terrain vague, on projeta d'établir un héliport, image parfaite de la tabula rasa. La façade arrondie du musée d'ethnologie faisait l'angle de la Königgrätzer Strasse et de la Prinz-Albrecht-Strasse. Au numéro 8 de cette dernière, la gestapo avait installé ses bureaux. Tout à côté, au numéro 9, l'hôtel Prinz-Albrecht était le quartier général de la Reichsfuihrung SS. Un musée consacré à la conservation des traces de sociétés en voie de disparition est donc devenu, ainsi que son voisinage, le siège de ceux qui organisèrent la disparition de communautés entières définies par 
l'appartenance politique, l'ethnie, la confession ou les comportements sexuels. Une fois la Seconde Guerre mondiale achevée, on voulut faire disparaître de la carte de Berlin-Ouest la moindre trace matérielle du passage des nazis. Il revint donc à quelques obstinés de ramener au jour les fondations des bâtiments détruits grâce à un travail de fouille qui pourrait être dit archéologique s'il ne concernait des vestiges vieux d'une quarantaine d'années seulement. C'est ainsi que sur l'emplacement du 8 Prinz-Albrecht-Strasse furent exhumés des murs et des bacs rectangulaires aux parois recouvertes de carreaux blancs: s'agissait-il de lieux de torture ou de cuisines, l'incertitude demeure, apparemment. Un petit bâtiment les recouvre et les protège. On y installa en 1987 une exposition temporaire qui porte le nom de Topographie des Terreurs et qui doit à l'affluence des visiteurs de ne pas avoir disparu. Godard témoigne de cela; son combatcontre est aussi un combat-pour.

L'explication est assez longue pour quelques plans (le carton et deux vues de l'espace nu et désert où régnèrent les maitres de l'Allemagne nazie et où souffrirent tant d'êtres humains); elle est nécessaire pour apprécier leur poids exact, mais pas pour ressentir leur charge émotionnelle. Au spectateur qui ne dispose pas de l'explication reste le texte du carton qui se suffit à luimême et qui irradie autour de lui. Jean Narboni, dans son essai sur le film, donne l'exemple du plan consacré au souvenir de deux jeunes gens, Hans et Sophie Scholl, exécutés pour avoir distribué des tracts anti-hitlériens: il s'agit d'une rose blanche, " [...] image d'une très grande beauté, à laquelle il me faut consacrer pas moins d'une page d'écriture pour essayer (telle une fleur japonaise trempée dans l'eau pour qu'elle s'épanouisse) d'en restituer, et sans doute diluer, la force d'évocation" (p. 55). Dans ce cas particulier, l'explication réside dans le fait que le groupe d'étudiants auquel appartenaient les deux jeunes gens portait le nom de "Rose blanche".

Dans Devant la recrudescence des vols de sacs à main, Serge Daney prend l'exemple de la guerre du Golfe et des étudiants de cinéma qui découvrent cette guerre à la télévision. L'absence de contrechamp dans la vision proposée du conflit est pour lui une absence de pensée sur le sujet, c'est-à-dire une absence de 
réflexion militante. Alors, il faut rappeler aux étudiants que le cinéma a fait "cadeau" à notre siècle d'instruments pour penser qui s'appellent montage, gros plan ou champ-contrechamp. C'est ainsi que Nuit et brouillard d'Alain Resnais a donné à ses spectateurs le " [...] sentiment de fierté à disposer, malgré tout, du cinéma, seul art capable de se tenir avec dignité au niveau d'un tel événement, d'informer et de former" (Daney et Roger, p. 132). On a pu dire que le travelling est affaire de morale et que le "point de vue" est quelque chose qui existe au cinéma. La formule de Godard est aux yeux de Daney " [...] un de ces truismes sur lesquels on ne reviendrait pas. Pas moi. En tout cas".

Dans les années soixante, Godard place Resnais très haut. Il a été impressionné par Nuit et brouillard et Hiroshima mon amour. L'idée d'un film sur les camps de concentration l'obsède. Il en parle dans Une femme mariée, mais il y revient dans Histoire(s) du Cinéma et dans Allemagne 90. Ce dernier film témoigne aussi de ce qu'Alain Badiou a qualifié de "désastre obscur", c'est-àdire de la déliquescence du monde communiste, avec ses corrélats, la chute du mur et la réunification de l'Allemagne. Ce pays n'a cessé d'occuper une position stratégique dans le glacis européen depuis la fin de la Deuxième Guerre mondiale, et le cinéma a témoigné de cette importance au travers d'œuvres aussi diverses que celles de Syberberg ou de Lars von Trier (mais on pourrait aussi bien mentionner Allemagne, année zéro de Rossellini, Berlin Express de Jacques Tourneur et bien d'autres films). Non seulement la guerre froide s'est-elle manifestée en Allemagne de façon plus nette qu'ailleurs, mais ce qui s'y est passé (les tortures, les camps...) renvoyait de façon plus aiguë à nos sociétés une image qu'elles condamnaient tout en l'illustrant chacune à son propre niveau et selon les circonstances historiques, avec des moyens plus ou moins modestes. Puis vint le jour où l'on entreprit d'effacer cette image en niant l'existence du référent qu'elle dupliquait. Ce pourquoi la guerre du Golfe fut elle aussi effacée, comme celle des Malouines transforma la mort des aviateurs en un jeu vidéo, et comme la réduction au silence des membres de la Fraction Armée Rouge par la société allemande a consisté en une disparition dans le blanc des cellules aseptisées où la lumière ne s'éteint jamais. 
Chaque fois que l'image se trouve atteinte, le cinéma a son mot à dire. En cette fin de siècle, aussi affaibli qu'il puisse être, il peut encore montrer des images et de la pensée, notamment les siennes indiscernables dans la texture même du siècle. C'est là le propos de Godard. Le cinéma peut penser le siècle ou plutôt, la fin de siècle. Il suffit qu'il soit entre les mains d'"observateurs scientifiques". Il construit cette analyse, ou cette réflexion, ce "rapport" dans une œuvre fragmentée mais qui possède à travers cette fragmentation sa cohérence propre. De même que Passion, Scénario du film Passion, Prénom Carmen, Je vous salue Marie, Grandeur et décadence d'un petit commerce de cinéma constituent les fragments d'un seul grand film, de même font $L e$ Dernier Mot, Puissance de la parole, Le Rapport Darty, Aria, L'Enfance de l'art et Allemagne 90 (d'un grand film à l'autre, il est d'ailleurs plus d'une passerelle et il n'est pas sûr qu'il ne s'agisse pas, tout compte fait, d'une seule œuvre dont l'unité tient moins à un auteur unique qu'à la démarche disséminante qui renvoie l'unité non pas à une réalité antérieure, précédant et réalisant par avance la "création", mais à un possible à venir). La plupart de ces œuvres (auxquelles il faudrait peut-être associer King Lear et Soigne ta droite) ont en commun d'être des commandes, d'avoir été réalisées entre 1987 et 1990 en vidéo ou sur support film. Des unes aux autres, des sons (tel cri d'oiseau, tel morceau musical, telle voix de chanteur, telle citation...) et des images (tel peintre, tels plans de foule - dès Lettre à Freddy Buache, Godard porte une grande attention aux déplacements de foule dans la rue - , tels mouvements, tels clignotements...) reviennent comme les signes d'une commune appartenance. Celle d'une pensée en exercice.

Que le cinéma soit une manière de penser, qu'il exerce cette capacité est en soi la manifestation d'une éthique. Il est plusieurs façons pour lui d'exprimer son rapport avec le monde de la pensée, qui sont en gros les mêmes que pour la littérature et que Pierre Macherey a énumérées (il suffit de remplacer le mot littérature par celui de cinéma, et l'expression texte littéraire par film):

Au niveau le plus élémentaire, le rapport de la littérature et de la philosophie est strictement documentaire: la philosophie affleure à la surface des œuvres de la 
littérature au titre d'une référence culturelle, plus ou moins travaillée, comme une simple citation, qui d'ailleurs, du fait de l'ignorance de leurs lecteurs et commentateurs, passe le plus souvent inaperçue. $\AA$ un autre niveau, l'argument philosophique remplit à l'égard du texte littéraire le rôle d'un véritable opérateur formel: c'est ce qui se passe lorsqu'il dessine le profil d'un personnage, organise l'allure générale d'un récit, voire en dresse le décor, ou structure le mode de sa narration. Enfin le texte littéraire peut encore devenir le support d'un message spéculatif, dont le contenu philosophique est souvent ramené sur le plan d'une communication idéologique (p. 11).

Le combat-contre renvoie plutôt à ce message spéculatif. Des références culturelles plus ou moins travaillées, Allemagne 90, comme tous les films de Godard, n'en manque pas (de Giraudoux à Rilke, de Goethe à Musil, Freud ou Benjamin). En revanche, l'argument philosophique comme "véritable opérateur formel" est moins visible, il demande de l'attention pour être décelé. Non pas que la pensée serait "cachée" et qu'il faudrait l'extraire de l'œuvre "comme un corps étranger " pour la présenter telle qu'en elle-même, mais parce que «ici le contenu n'est rien en dehors des figures de sa manifestation" (p. 197).

Il est évident que Godard joue sur la diffraction des signifiés : l'énumération qui est faite à un moment des prénoms féminins (Dora, Frieda, etc.) évoque d'abord, à l'intérieur de l'œuvre du réalisateur, celle, ludique, que l'on trouve dans la scène des cartes postales des Carabiniers. L'on peut aussi penser, comme Jean Norboni se souvenant de Nuit et brouillard, à ces usines des camps de concentration qui portaient des noms de jeunes filles. On peut songer encore au court récit d'Anna Saghoro intitulé L'Excursion des jeunes filles qui ne sont plus, requiem dédié à 15 jeunes filles disparues tragiquement sous la domination hitlérienne ": "Jeunes filles sans uniforme", dit un carton.

L'une des figures par lesquelles se manifeste le contenu est la différence des images de 1990, en couleurs par rapport à celles du passé, généralement en noir et blanc (à l'exception des plans des Quatre Cavaliers de l'Apocalypse de V. Minnelli), prises sur un écran vidéo et au rythme retravaillé, avec des ralentissements, 
des arrêts sur image, des retours en arrière, des superpositions, etc. Godard a mis au point ce traitement particulier des images du passé (qui peuvent être aussi des peintures) dans ses Histoires(s) du Cinéma en 1988, mais c'est le résultat d'un travail d'une dizaine d'années. Un combat s'engage entre ces images, qui vise à s'emparer de la force de celles du passé (qu'il s'agisse de vues documentaires, d'extraits de La Passagère de Munk, des Hommes le dimanche de Siodmak, Wilder, etc., ou d'Au bord de la mer bleue de Boris Barnet) pour se l'approprier et revigorer celles du présent, mortellement menacées par l'industrie américaine.

Il suffit de regarder successivement Le Rapport Darty (avec le rôle qu'y joue Marx, et le combat de chevaliers venus de Perceval de Rohmer...), Histoire(s) du cinéma (avec les extraits des Quatre Cavaliers d'Apocalypse, de La Passagère, d'Alexandre Nevsky, de La Mort de Siegfried ou de Mabuse) et Allemagne 90 pour s'apercevoir de la continuité d'une œuvre à l'autre. La référence à Don Quichotte dans Allemagne 90 renvoie au film inachevé de Welles (dont il est question dans Histoire(s)), mais sans doute aussi à celui, terminé, de Pabst, et à coup sûr au Pont $d u$ Nord de Jacques Rivette, avec ses dragons représentés par les armatures métalliques et les bras d'acier des grues: "Les dragons de notre vie ne sont que des princesses qui attendent de nous voir beaux et courageux", etc. Lemmy Caution, qui est resté 30 ans à tourner en rond dans les problèmes de la guerre froide, a battu les records de la Belle au bois dormant dans sa chambre froide, vraie concession à perpétuité. Il fait penser à Siméon du désert isolé sur sa colonne. À propos du film de Buñuel, Serge Daney fait l'hypothèse que "le Mal a gagné. Et ça m'amuse beaucoup d'imaginer Buñuel comme un journaliste abonné à une sorte de fil AFP secret de la métaphysique chrétienne et qui, un jour, lit une dépêche où il est dit qu’après des péripéties complexes, le Mal a gagné la guerre. Alors, il dit à Siméon : pendant que tu faisais l'imbécile là-haut sur ta colonne, tout s'est joué ailleurs, sans toi, et tu as perdu" (Daney et Roger, p. 135). Les Brigands triomphent, dirait Badiou reprenant Robespierre; et Lemmy Caution, en pensant à Schiller! "Les Brigands, j'ai connu ça toute ma vie. " L'escalier qu'emprunte Lemmy 
Caution au début du film (et qu'il renonce à monter, appelé qu'il est à chercher la route de l'Ouest) évoque celui de Muriel d'Alain Resnais au sommet duquel était enclos le secret d'une guerre: le retour en arrière du dernier agent secret (le sous-titre de Muriel pourrait être celui d'Allemagne 90, le temps d'un retour) signifie tout simplement la fin de la guerre froide, l'Ouest a vaincu par neuf à zéro. L'agent n'était pas dans le secret.

Il faut revenir au titre Allemagne 90 car il est le lieu d'un combat-entre très godardien. Godard a parlé du redoublement du 9 dans 1990 (en inversant l'un, on obtient 69, une position répertoriée; si on entrecroise les deux chiffres se dessine alors la figure de l'infini)... Que ce titre renvoie à Rossellini, c'est l'évidence même. Mais aussi au Zéro et l'infini, à Zéro de conduite, et encore à l'alpha et à l'oméga (d'où Alphaville), au neuf opposé à l'ancien (L'Ancien et le Nouveau), à Nouvelle Vague, d'où toutes les allusions au dernier, Le Dernier des Hommes, le dernier documentaire de l'UFA, la fin de l'Allemagne, "finis Germaniae"... Mais " $f$ nis", c'est la frontière (celle au-delà de laquelle viennent les fantômes), ce lieu-fantôme que ne trouve pas le dernier espion. "Which Way to the Front?" demandait Jerry Lewis, question que Lemmy Caution traduit par Which Way to the West? "Hélas oui, c'est par là l'Occident ", à quoi Lemmy Caution ajoute: "Pauvre de moi..." "Deux âmes, hélas, habitent ma poitrine", Hélas pour moi se trouve programmé dès Histoire(s) du Cinéma et Allemagne 90. La loi des séries et l'association semi-libre permettent de définir un champ ouvert à tous les combats-entre.

Dans Alphaville, Lemmy Caution venait des mondes extérieurs, puis y retournait. Dans Allemagne 90, son voyage le conduit de l'Est vers l'Ouest, d'un monde un peu triste, désuet et vide vers les "incroyables saloperies" qu'offrent les villes occidentales. "Test the West!» De la vacuité au trop-plein (où l'on ne trouve pas ce qui correspond aux besoins élémentaires). La solitude à deux. Réunis, quelle fiction: la réunification, avec un $\mathrm{A}$ pour Alphaville. Le mot Aeroflot ne peut même plus signifier un départ vers un ailleurs possible (ou probable? improbable?). La parole est atteinte dans ses forces vives. L'acheminement vers elle ne conduit que dans la ville où l'on châtre la Bible, où l'on efface les mots. Ce qui justifie le mot final: "Ah! les salauds!» Ces 
chemins ne mènent donc nulle part. La parole est un autre lien qui relie les œuvres de Godard, du dernier mot du philosophe Valentin Feldman à la puissance matérielle de la parole et au verbe incarné dans Hélas pour moi, en passant par les mots d'Armide de Lully et par ceux de Hugo dans Les Misérables. Le souvenir d'Alphaville et de la voix du robot, c'est-à-dire de la parole désincarnée, est présent dans Le Rapport Darty et Hélas pour moi.

Daney conclut son interprétation risquée du film de Buñuel en écrivant: "Comme dit mystérieusement Godard: l'Histoire est seule." Cela, Godard le dit dans Histoire(s) du Cinéma. Le vrai sous-titre d'Allemagne 90 est: "Un état et des variations" et le film est ponctué de cartons: "Histoire de la solitude" (deux fois), "Une histoire seule" (trois fois), "Solitude de l'Histoire" (deux fois). Le commentaire dit que le rêve de l'État est d'être seul, alors que celui des individus est d'être deux. Le carton « $\mathrm{Ne}$ te fais pas de mal, nous sommes tous encore ici" est déjà dans Histoire(s) du Cinéma. Godard a donc mis au point une "méthode" pour introduire à une véritable Histoire du Cinéma: le mot Histoire étant au singulier ou au pluriel, car l'un et l'autre se dit ou se disent, et cette Histoire est une multiplicité où entrent en ligne de compte Allemagne 90 comme Les Enfants jouent à l'Union soviétique, ou L'Enfance de l'art. Un état: la solitude, - et ses variations: à travers l'Histoire du Cinéma, celle de l'Allemagne actuelle, mais aussi à travers la "solitude essentielle" dont parle Rilke à un jeune poète ${ }^{2}$, cette solitude que commente Maurice Blanchot dans sa relation à la dissimulation. Le carton bilingue "Là où c'était je dois advenir " fait référence à Freud, certes, mais ne peut-on y voir une condensation de ces lignes de L'Espace littéraire?

Quand les êtres manquent, l'être apparaît comme la profondeur de la dissimulation dans laquelle il se fait manque. Quand la dissimulation apparaît, la dissimulation, devenue apparence, fait "tout disparaître ", mais ce "tout a disparu" fait encore une apparence, fait que l'apparence a désormais son point de départ dans " tout a disparu ". "Tout a disparu" apparaît. Ce qu'on appelle apparition est cela même: est le "tout a disparu" devenu à son tour apparence. Et l'apparition dit précisément que quand tout a disparu, il y a encore quelque 
chose: lorsque tout manque, le manque fait apparaître l'essence de l'être qui est d'être encore là où il manque, d'être en tant que dissimulé... (p. 265).

Reconsidérons Allemagne 90. Un État, l'Allemagne, "quand tout a disparu" ("Il vous dit adieu, Mademoiselle! »), la dissimulation qui apparaît (quoi de plus dissimulé qu'un agent secret a dû se dire Jean-Luc Godard), le moment de l'apparition que traque le cinéaste (l'image viendra au temps de la résurrection)... Cela a un très beau nom, cela s'appelle l'aurore - en allemand morgenröthe, n'est-ce pas monsieur Godard? "On désapprend le silence quand, aussi longtemps que lui, on a été taupe, on a été seul..." (Nietzsche, p. 13).

Il est possible que le "questionnement éthique" ou que "les termes éthiques initiaux du combat contre l'indifférence" tels que je les décris apparaissent "trop ténus". C'est qu'il faut comprendre que la position éthique définie à travers l'œuvre de ce cinéaste est celle d'un acteur politique. Godard, comme Michel Foucault, selon la définition de Paul Veyne (Le Dernier Foucault et sa morale), est un "guerrier": "Un guerrier est un homme qui peut se passer de la vérité, qui ne connaît que des partis pris, le sien et celui de l'adversaire, et qui a l'énergie suffisante pour se battre sans devoir se donner raison pour se rassurer."

Si le premier acte consiste pour Godard à se souvenir, c'est que "la mémoire est toujours de la guerre" comme le disait Péguy en opposant le chroniqueur à l'historien. Il n'y a pas de vérité morale transcendante qui justifie ce combat-contre. Il serait erroné de confondre la loi morale et l'Éthique (au sens de Spinoza). Celle-ci, "[...] typologie des modes d'existence immanents, remplace la Morale, qui rapporte toujours l'existence à des valeurs transcendantes" (Deleuze, 1981, p. 35). La liberté résulte d'une pratique effective. Les termes "éthiques" ne sont pas au départ mais devant. Ils sont l'horizon du combat (ou si l'on préfere de l'action) et non sa condition. Il s'agit pour l'individu de se donner les moyens de vaincre. Godard, dans Allemagne 90, décrit une situation, il dresse une carte, il détermine les positions de l'ennemi, la société internationale de contrôle qui s'est mise en place ces dernières années et dans laquelle il voit certainement une puissance triste fabriquant des esclaves. 
L'Allemagne est pour cela un bon lieu d'observation en Europe. C'est dans l'actualité, présent, passé et futur repliés les uns sur les autres, qu'il faut sans cesse épouser les forces du devenir: ce qui revient à renforcer sa propre puissance, ce qui s'appelle donner corps à une "éthique", ce qui exclut toute ratiocination, toute idée de "questionnement".

\section{Université de Paris III (Sorbonne Nouvelle)}

\section{NOTES}

1 Anna Saghoro, L'Excursion des jeunes filles qui ne sont plus (Paris: Ombres, 1993). La première édition allemande date de 1946 et la première publication en France, de 1956.

2 Le film cite plusieurs fois les Lettres à un jeune poète, dont: "La solitude est une, elle est par essence grande et lourde à porter."

\section{OUVRAGES CITÉS}

Blanchot, Maurice. L'Écriture du désastre. Paris: Gallimard, 1980.

Blanchot, Maurice. L'Espace littéraire. Paris: Gallimard, 1955.

Daney, Serge. L'exercice a été profitable, Monsieur. Paris: P.O.L., 1993.

Daney, Serge et Roger, Philippe. Devant la recrudescence des vols de sacs à main, cinéma, télévision, information (1988-1991). Paris: Aléas, 1991.

Deleuze, Gilles. Critique et clinique. Paris: Minuit, 1993.

Deleuze, Gilles. Spinoza, philosophe pratique. Paris: Minuit, 1981.

Jacob, André. "Éthique». Encyclopédie philosophique universelle, vol. 2, t. 1. Les Notions philosophiques. Paris: P.U.F., 1990.

Macherey, Pierre. À quoi pense la littérature. Paris : P.U.F., 1990.

Narboni, Jean. "Tous les autres s'appellent Meyer". Trafic, nº 3 (été 1992).

Nietzsche, Friedrich. Aurore. Paris: Gallimard, 1970. 